\title{
Video Article \\ High-definition Fourier Transform Infrared (FT-IR) Spectroscopic Imaging of Human Tissue Sections towards Improving Pathology
}

\author{
Hari Sreedhar* ${ }^{1}$, Vishal K. Varma ${ }^{* 1}$, Peter L. Nguyen ${ }^{2}$, Bennett Davidson ${ }^{3,4}$, Sanjeev Akkina ${ }^{5}$, Grace Guzman ${ }^{2}$, Suman Setty ${ }^{2}$, Andre Kajdacsy- \\ Balla ${ }^{2}$, Michael J. Walsh ${ }^{2}$ \\ ${ }^{1}$ Department of Bioengineering, University of Illinois at Chicago \\ ${ }^{2}$ Department of Pathology, University of Illinois at Chicago \\ ${ }^{3}$ Department of Biological Sciences, University of Illinois at Chicago \\ ${ }^{4}$ Department of Chemistry, University of Illinois at Chicago \\ ${ }^{5}$ Department of Nephrology, University of Illinois at Chicago \\ *These authors contributed equally
}

Correspondence to: Michael J. Walsh at walshm@uic.edu

URL: https://www.jove.com/video/52332

DOI: doi:10.3791/52332

Keywords: Medicine, Issue 95, Spectroscopy, Imaging, Fourier Transform, Pathology, Cancer, Liver, Kidney, Hyperspectral, Biopsy, Infrared, Optics, Tissue

Date Published: 1/21/2015

Citation: Sreedhar, H., Varma, V.K., Nguyen, P.L., Davidson, B., Akkina, S., Guzman, G., Setty, S., Kajdacsy-Balla, A., Walsh, M.J. High-definition Fourier Transform Infrared (FT-IR) Spectroscopic Imaging of Human Tissue Sections towards Improving Pathology. J. Vis. Exp. (95), e52332, doi:10.3791/52332 (2015).

\section{Abstract}

High-definition Fourier Transform Infrared (FT-IR) spectroscopic imaging is an emerging approach to obtain detailed images that have associated biochemical information. FT-IR imaging of tissue is based on the principle that different regions of the mid-infrared are absorbed by different chemical bonds (e.g., $\mathrm{C}=\mathrm{O}, \mathrm{C}-\mathrm{H}, \mathrm{N}-\mathrm{H}$ ) within cells or tissue that can then be related to the presence and composition of biomolecules (e.g., lipids, DNA, glycogen, protein, collagen). In an FT-IR image, every pixel within the image comprises an entire Infrared (IR) spectrum that can give information on the biochemical status of the cells that can then be exploited for cell-type or disease-type classification. In this paper, we show: how to obtain IR images from human tissues using an FT-IR system, how to modify existing instrumentation to allow for high-definition imaging capabilities, and how to visualize FT-IR images. We then present some applications of FT-IR for pathology using the liver and kidney as examples. FT-IR imaging holds exciting applications in providing a novel route to obtain biochemical information from cells and tissue in an entirely label-free non-perturbing route towards giving new insight into biomolecular changes as part of disease processes. Additionally, this biochemical information can potentially allow for objective and automated analysis of certain aspects of disease diagnosis.

\section{Video Link}

The video component of this article can be found at https://www.jove.com/video/52332/

\section{Introduction}

IR spectroscopy has been an analytical tool available in some form since the 1930s; however, it is only been within the last decade that the area of tissue imaging with FT-IR has exploded. The advances in FT-IR for tissue imaging have been driven in a large part by three key developments: 1) increased speed of data acquisition due to the availability of large Focal Plane Array (FPA) detectors which typically have thousands of IR sensitive detectors ${ }^{1,2}, 2$ ) development of advanced processing algorithms and computational power to handle large hyperspectral data sets ${ }^{3}$, and 3) modeling of FT-IR imaging systems to maximize spatial resolution ${ }^{4,5}$. There have been numerous high quality and very extensive articles reviewing the field of FT-IR spectroscopy recently ${ }^{6-16}$, in addition to a Nature Protocols paper that details the steps to obtain point spectra or maps from tissues ${ }^{17}$. In this paper, we will focus on the protocol to obtain images of tissues using a $128 \times 128 \mathrm{FPA}$ detector in a modified FT-IR system with high-definition capabilities.

FT-IR imaging has long been suggested to be a potentially desirable tool for cell and tissue imaging due to the ability to obtain images in which every pixel has a wealth of biochemical information. FT-IR imaging is based on the principle that different biomolecules in a sample will quantitatively absorb different regions of the mid-infrared; this allows for the derivation of a 'biochemical fingerprint'. This fingerprint had been shown in many studies to alter between different cell types and disease states. Unlike in conventional pathology practice where stains and immunohistochemical markers need to be used to visualize and identify cell types and tissue structures that are used to guide diagnosis and treatment options, the images from FT-IR are formed based on the inherent biochemistry of the tissue. The current technique of staining tissue for diagnosis is time-consuming, destructive, laborious, and requires subjective expertise of the pathologist, whereas FT-IR offers the potential to make this process rapid, non-destructive, highly automated, and more objective. In addition, FT-IR provides a novel route to obtaining additional biochemical information that may not be readily accessible using conventional staining techniques. 
One of the most exciting advances in recent years has been the availability of high resolution imaging approaches that can now allow for the visualization and characterization of cell types and tissue structures that are critical for comprehensive disease diagnosis. One of these techniques is Attenuated Total Reflectance (ATR) FT-IR which incorporates a solid immersion lens (SIL) of a high refractive index which allows for high resolution imaging ${ }^{18}$, with many very exciting studies showing its applications ${ }^{19-25}$. In addition, it was recently demonstrated that the increased spatial resolution associated with ATR imaging can allow for the visualization and classification of endothelial and myoepithelial cells in breast tissue which form a key component of breast cancer diagnosis ${ }^{26}$. While ATR imaging is very useful, this technique requires the SIL to make contact with the tissue to form FT-IR images; therefore, its use is somewhat limited for tissue pathology where large regions of tissues must be quickly imaged.

A second approach was demonstrated by coupling a high magnification objective to an existing FT-IR system that uses a synchrotron as a bright source of IR, it is possible to fully illuminate an FPA and image with an effective pixel size of $0.54 \times 0.54 \mu \mathrm{m}$. This allowed for us to visualize key structures in breast and prostate tissues that were not resolvable using conventional FT-IR systems ${ }^{4}$. While these dramatic increases in IR image spatial resolution were exciting, its use remained limited due to requiring a synchrotron. Subsequently, an optimal system was designed that could also allow for high-definition imaging capabilities with a $1.1 \times 1.1 \mu \mathrm{m}$ pixel size without the requirement of a synchrotron source but rather using a traditional globar IR source ${ }^{5}$. In this article, we show how to modify an existing commercial FT-IR imaging system to allow for diffraction limited IR imaging of tissues with an acceptable signal to noise ratio using multiple IR objectives (15X, 36X, and 74X). The effective pixel size with the three objectives is $5.5 \times 5.5 \mu \mathrm{m}(15 \mathrm{X}), 2.2 \times 2.2 \mu \mathrm{m}(36 \mathrm{X})$ and $1.1 \times 1.1 \mu \mathrm{m}(74 \mathrm{X})$. We then give some examples of the importance of the gains in spatial resolution for disease detection in liver and kidney biopsies ${ }^{27}$.

\section{Setting up an FT-IR Microscope and Acquiring Tissue Images}

1. Section a formalin-fixed paraffin-embedded tissue block at $4 \mu \mathrm{m}$ thickness on to an IR compatible slide using a microtome. Alternately, section a liquid nitrogen frozen tissue at $4 \mu \mathrm{m}$ thickness onto an IR compatible slide using a cryostat.

NOTE: Typically, a serial section will also be acquired on a glass slide and stained with a special (such as Haematoxylin and Eosin (H\&E)) or immunohistochemical stain. In addition, cultured cell lines can be grown on IR compatible slides. IR compatible slides can be IR reflective slides for reflection mode imaging (e.g., MirrlR slide, gold) or IR transparent slides for transmission mode imaging (e.g., $\mathrm{BaF}_{2}$, $\left.\mathrm{CaF}_{2}\right)$.

2. Purge the FT-IR microscope and spectrometer using either dry air or dry nitrogen gas to remove atmospheric water from the system. Wait at least 45 min before imaging to ensure a thorough purge.

3. Cool both the FPA detector (to $79 \mathrm{~K}$ ) and the internal MCT detector in the FT-IR microscope using liquid nitrogen. Cool the detectors approximately every $6 \mathrm{hr}$.

CAUTION! Liquid nitrogen is a cryogenic fluid and can cause cold burns, frostbite and in enclosed spaces asphyxiation.

4. Mount the sample slide on the microscope stage for FT-IR imaging.

5. Ensure the visible light is "ON" and then either using the binoculars or the sample capture program (which provides a real-time visible image of the tissue), focus on the sample.

6. Open the bundle software package such as resolutions pro and click 'Collect', then click 'Diagnostics' and then select 'Align Spectrometer'. Ensure that the beam path to the spectrometer internal detector is not obstructed.

7. Click on 'Imaging Setup'.

8. In the 'Electronics' tab, select the appropriate 'Speed', 'Under Sampling Ratio (UDR)' and 'Filters' settings depending on the system. NOTE: In this example, the setting is Speed $=2.5 \mathrm{KHz}$, UDR $=4$ and Filters $=$ None.

9. In the 'Optics' tab ensure that 'Mid-IR source', 'Open aperture' and ' $100 \%$ beam attenuator' are selected.

10. To calibrate the system, in the 'Optics' tab, select 'Detector' as 'Ground', 'Microscope Detector' as 'Left', and then select either 'Transmittance' or 'Reflectance' under 'Optics mode' depending on the type of slide being used.

11. Click Setup, which will open up the 'Lancer Control' window. NOTE: For reflection mode follow instructions in 1.12 and 1.13 and for transmission mode follow instructions in 1.14 to 1.16 . Continue from 1.17 for both reflection and transmission.

12. For reflection mode, in 'Lancer Control' click on 'Raw'. Using the stage control joystick and watching the live view of the FT-IR interferogram image (top right image), move to a clean area on the slide.

13. Adjust the integration time to approximately 8,000 counts. Next, move the stage to find a piece of tissue with structure, preferably the edge of a tissue, and perfect the focus of the image. Change 'Warmth' and 'Contrast' options to help form an image to improve focusing, this will have no effect on the IR data. Continue from 1.18

14. For transmission mode, in 'Lancer Control' click on 'Raw'. Using the stage control joystick and watching the live view of the FT-IR interferogram image (top right image), move to a clean area of the slide.

15. Adjust the integration time to approximately 8,000 counts.

16. Adjust the bottom focusing/condenser objective to increase the number of counts to its maximum. Watch the shape of the bottom right image in Lancer Control to ensure that it is Gaussian in appearance and relatively uniform. Adjust the integration time again to approximately 8,000 counts.

NOTE: If any of the pixels in the FT-IR interferogram image turn white, reduce the integration time.

17. Move the stage to find a piece of tissue with structure, preferably the edge of a tissue and perfect the focus of the image. Optionally, change the warmth and contrast options to help form an image to improve focusing, but will have no effect on the IR data.

18. Using the stage control joystick, move to a clean area of the slide. Press the 'Calibrate' button. Ensure the 'Out Of Range (OOR)' value is less than 50 and the difference between the 'High Flux' and 'Low Flux' counts is at least 4,000 counts.

19. Select 'OK' twice. In the optics tabs select; 'Detector' = 'MCT', 'Microscope Detector' = 'Right' and then click 'Setup'. At this point there will be a FT-IR interferogram on the screen. Click on 'Find Centerburst'. Click 'Okay'.

20. In the 'Optics' tab, reselect 'Detector' = 'Ground', 'Microscope Detector' = 'Left' and select 'Setup'.

21. In Lancer Control - ensure the image is still on a clean area and click 'Calibrate' again. Click 'Okay'. 
22. Next, collect a background FT-IR image to correct for absorbance from the atmosphere, system and slide. Go to the 'Electronics' tab and select an appropriate spectral resolution, typically of $4 \mathrm{~cm}^{-1}$ or $8 \mathrm{~cm}^{-1}$ for tissue.

23. Go to the 'Background' tab and type 128 in 'Scans to co-add'. Select the 'New File...' button and place the background file in appropriate folder. Check the 'Imaging' tab to ensure mosaicing is 1 by 1 . Click 'Background', wait for the scan to finish and confirm where to save the file. Click a region on the background FT-IR image and check the spectrum.

24. To take a large mosaic image of the sample, select the 'Imaging' tab and insert the number of $X$ and $Y$ frames for the mosaic size you wish to acquire.

25. Click 'Setup' and in Lancer Control use the live IR view to find the area of interest. If taking a mosaic, center the live feed in the middle of the area of interest. Click 'Okay'. Go to the 'Electronics' tab and type the number of scans to co-add (typically a value between 2 and 16 scans for tissue). Click 'Scan'.

26. Check the collected FT-IR image on the screen to ensure it looks focused. Click on the image to bring up an IR spectrum from that location and check that it looks to have an acceptable signal to noise. Acquire a visible image of the sample.

27. Save the image and export it to the required format, such as ENVI-IDL.

\section{Adapting an FT-IR Microscope for High-definition Capabilities}

NOTE: Most FT-IR systems are equipped with an objective approximately $15 \mathrm{X}$ magnification and 0.5 numerical aperture (NA). To image in highdefinition mode, an IR compatible 36X or 74X objective can be used to give diffraction limited imaging capabilities.

1. Remove the system's $15 X$ objective by unscrewing counter clockwise.

2. Screw in either the $36 \mathrm{X}$ or $74 \mathrm{X}$ objective in its place. Align the objective prior to use. Use lens extension tubes to bring the objective close enough to the sample.

3. Place tissue under the new objective and focus using visible light using either the binoculars or Sample Capture program. NOTE: High-definition imaging must be done in transmission mode. The very close working distance of these objectives (1-2 $\mathrm{mm})$ and very shallow depth of focus requires focusing slowly and carefully, taking time to lower the objective without touching the sample.

4. Follow instructions from 1.6 to 1.11 . NOTE: Due to being significantly more difficult to focus and much less light reaching the detector, the following protocol is adjusted based on 1.14 .

5. In transmission mode, click on 'Raw'. Using the stage control joystick and watching the live view of the FT-IR interferogram image (top right image), move to the tissue and focus (ideally on an edge).

NOTE: This may be difficult, therefore adjust the 'Warmth' and 'Contrast' options to help form an image to improve focusing (these options have no affect on the IR data).

6. Once focused, using the stage control joystick and the live IR view move to a clean area of the slide. Adjust the integration time to approximately 8,000 counts. Adjust the bottom focusing objective to increase the number of counts to its maximum. Adjust the integration time again to approximately 8,000 counts.

7. Move the stage to find a piece of tissue with structure, preferably the edge of a tissue, and perfect the focus of the image.

8. If mosaicking an image at high resolution, adjust the stage to allow correct movement required across distances for accurate mosaicing. To change the stage distance settings, go to 'Setup stage' in Lancer Control and adjust the horizontal and vertical alignment settings to allow for correct mosaicking.

9. Using the stage control joystick move to a clean area of the slide. Press the 'Calibrate' button. Ensure the Out Of Range (OOR) value is less than 50 for both $36 \mathrm{X}$ and $74 \mathrm{X}$ objectives. Ensure the difference between the High Flux and Low Flux values is at least 1,000 counts for the $74 \mathrm{X}$ objective and 2,000 counts for the $36 \mathrm{X}$ objective.

10. Continue from 1.19 to 1.27 . The number of scans in 1.23 and 1.25 will need to be adjusted due to reduced signal to approximately 256 scans for the background image and 16 to 128 scans for the tissue image.

\section{Visualizing and Classifying IR Spectral Datasets}

NOTE: In this section, we will discuss how to visualize and extract data from spectral images using geospatial image processing and analyzing software such as ENVI+IDL, however the process is very similar for any alternate software such as MATLAB, free software such as CytoSpec, or the instrument developer's own software. There are a few different spectral processing techniques that may be performed on the IR data.

1. Open geospatial image processing and analyzing software and load the IR data file.

2. Apply a baseline correction algorithm to the IR data by selecting "Spectral tools" and scroll down and click "Absorbance spectra". Observe a pop-up menu and select "Baseline correction"

NOTE: Baseline correction will remove a baseline offset slope from the data due to scattering

3. Perform Spectral normalization, by ratioing the data to a certain spectral peak (typically Amide I $\left(1,650 \mathrm{~cm}^{-1}\right)$ or Amide II $\left.\left(1,550 \mathrm{~cm}^{-1}\right)\right)$ or area under a defined region of the spectra. Do this by selecting "Normal spectra" under the "Absorbance Spectra" menu options.

NOTE: Spectral normalization is performed so that any differences in spectra are real biochemical differences and not due to thickness or density differences between samples.

4. Use a noise reduction algorithm to remove noise from the spectra if necessary ${ }^{28}$

NOTE: There are multiple approaches available by which baseline correction, spectral normalization and noise reduction can be achieved, with most software having automated algorithms built in. In addition, there are an emerging number of approaches that will correct for spectral aberrations, which have been discussed in detail ${ }^{29-42}$; however, the community does not yet agree on which of these are needed.

5. Observe a list of all the IR frequencies collected within the image (typically of a spectral range from 900 to $4,000 \mathrm{~cm}^{-1}$ ). Click on the frequencies that correspond to specific biomolecules to observe an image of the tissue at the selected frequency.

1. For example, click on $1,650 \mathrm{~cm}^{-1}$ band to observe the distribution of proteins in the sample. Alternatively click on $1,080 \mathrm{~cm}^{-1}$ band to observe the distribution of nucleic acids in the sample. Use wavenumber $1,650 \mathrm{~cm}^{-1}$. 
6. Create additional images by computing spectral peak height ratios, peak area ratios etc. that will allow for visualization of different biomolecular components. Click on 'Spectral Tools' and then select either 'Peak Height Ratios' or 'Calculate Image Area' to create images.

7. Scan the corresponding adjacent tissue section which is stained using a separate whole slide imager system that captures brightfield images. Alongside the IR image, bring up the digital image of the stained tissue with a visible image program.

8. Right click on the image at a region of interest and select ' $Z$ profile'. This will give the spectral information at the selected pixel.

9. Look at the IR spectra of multiple pixels from multiple classes, such as comparing cell types, disease states. Mark specific pixels on the image using the 'Regions Of Interest' (ROI) tool. To perform this, right click on the image and select 'ROI tool'. Create the classes that are to be labeled, for example normal, dysplasia, and cancer classes. Then select, 'ROI type' - 'Point'. Then select the class to select pixels for and draw on the appropriate pixels on the IR image. Derive the average spectra for each of the classes using the 'Average ROI' tool.

10. Compare derived spectra by plotting in graphing software.

\section{Representative Results}

FT-IR imaging allows for the derivation of IR images of tissue that can give different contrasts depending on the IR frequency of interest. In addition, in an IR image, every pixel comprises of the entire IR spectrum, with different peaks corresponding to different biomolecules which can give information about the biochemical properties of cell types or disease states (Figure 1). Here, we have shown how to compare spectral signatures between classes, however more advanced automated classification is possible using additional algorithms ${ }^{3,43-50}$, such as Bayesian classification, Random Forests, Artificial Neural Networks, and Hierarchal Cluster Analysis can be performed on the data. Supervised classification approaches will allow for the construction of a classifier that can be trained to allow for automated recognition of cell types or disease states. Unsupervised classification approaches can be used to look for naturally occurring differences in tissue or cells due to biochemical variance.

FT-IR instrumentation has evolved over the past few decades, from measuring in a single point/mapping mode using IR opaque apertures to imaging mode using Cassegrain objectives, using either an illuminating objective coupled with a collecting objective in transmission-mode or a single objective that both illuminates and collects in reflection-mode (Figure 2). It was recently demonstrated that the collecting objective in transmission mode could be switched out for a higher magnification and numerical aperture objective to allow for diffraction limited IR imaging, that leads to substantial increases in the spatial resolution of collected IR images ${ }^{4,5}$. The advances in spatial resolution for tissue imaging have been of critical importance as we can now identify cell types and tissue structures, for example the functional units of the kidney, the glomeruli, using adapted in-house FT-IR systems (Figure 3).

High-definition FT-IR imaging allows for detailed images of tissues to be examined to identify abnormal regions and to identify biochemical differences between different cell types. In a liver tissue core, it is possible to visualize hepatocytes and regions of infiltrating fibrosis that divides two distinct areas of dysplasia and non-dysplastic cirrhosis (Figure 4). We are working to exploit this towards making automated diagnostic tools for use in difficult cases of liver disease.

Importantly, the increased spatial resolution can now allow us to isolate specific structural features that may be chemically modified by disease before histological changes are apparent. For example, we are focused on identifying biochemical changes in kidney glomerular structures such as the Bowman's capsule, mesangium, glomerular basement membrane and tubular basement membrane, before changes identified by the pathologist can be observed (Figure 5). In particular, we are interested in identifying changes associated with the progression of diabetic nephropathy and chronic rejection in transplant patients, where current techniques fail to identify changes in an early enough fashion for successful intervention. 

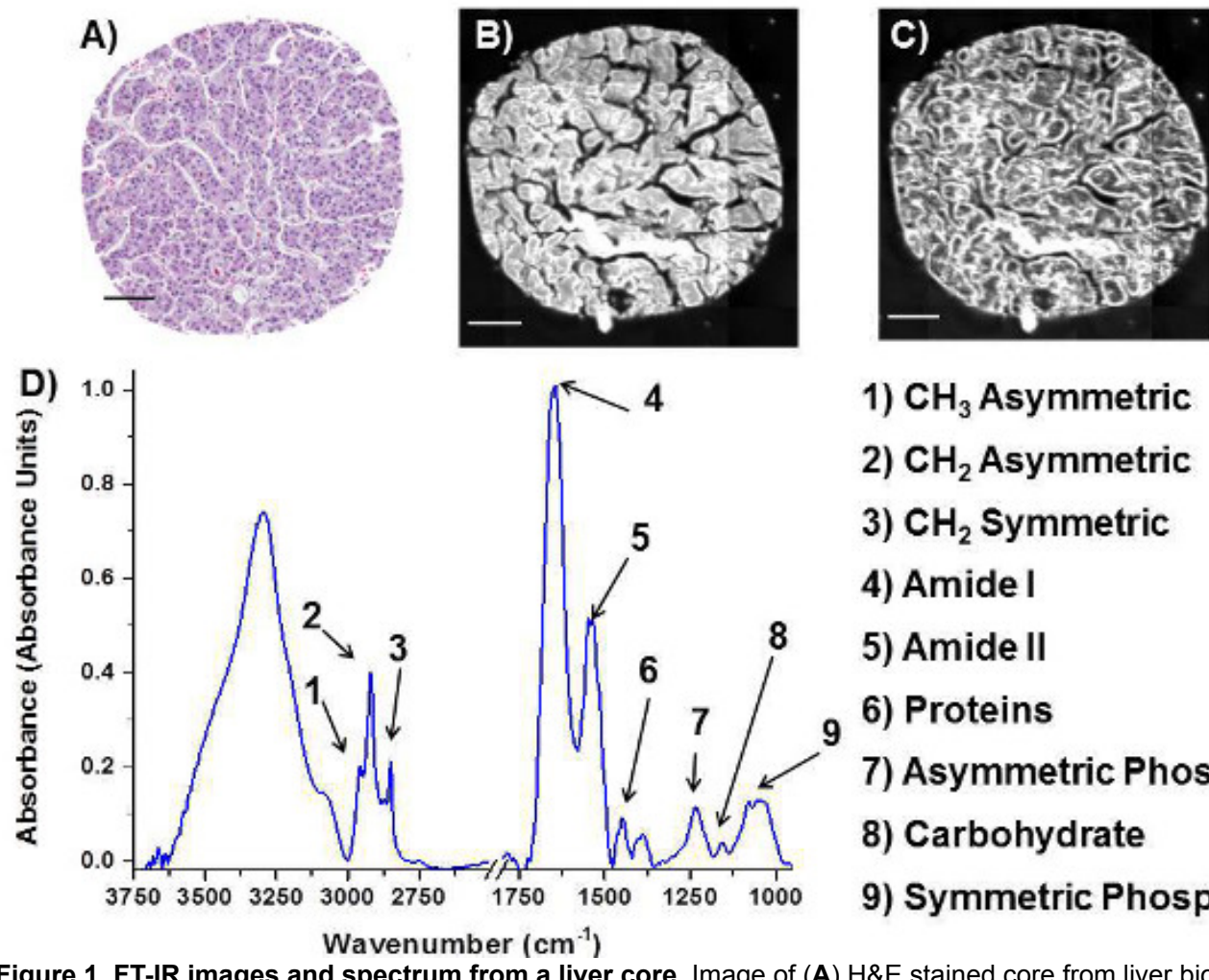

1) $\mathrm{CH}_{3}$ Asymmetric

2) $\mathrm{CH}_{2}$ Asymmetric

3) $\mathrm{CH}_{2}$ Symmetric

4) Amide $I$

5) Amide II

6) Proteins

7) Asymmetric Phosphate

8) Carbohydrate

9) Symmetric Phosphate

Figure 1. FT-IR images and spectrum from a liver core. Image of (A) H\&E stained core from liver biopsy and images of IR absorbance of serial section of the same core at (B) $3,286 \mathrm{~cm}^{-1}$ and (C) $2,603 \mathrm{~cm}^{-1}$, which highlighted different structural features. (D) Typical IR spectrum of tissue, with important peaks labeled. Scale bar $=100 \mu \mathrm{m}$. Please click here to view a larger version of this figure.

\section{A) Transmission-mode}

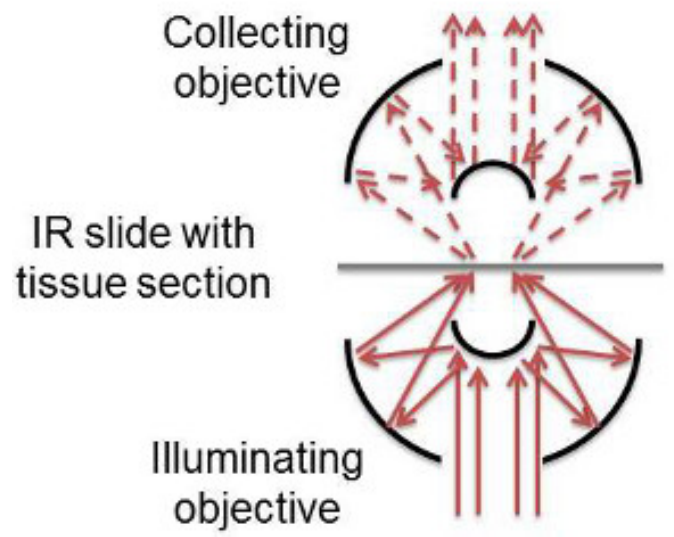

B) Reflection-mode

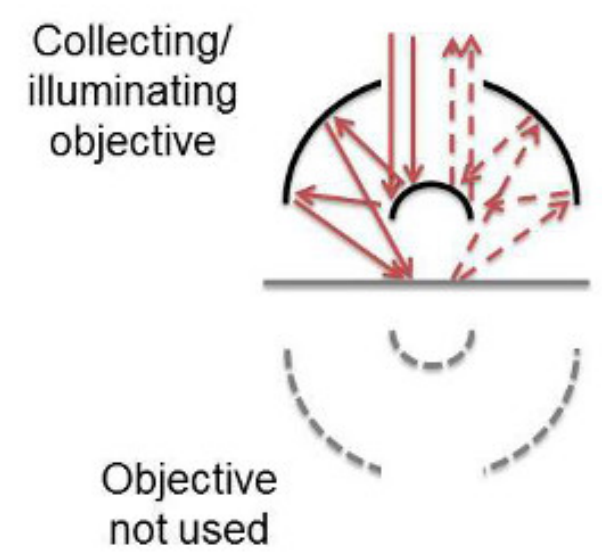

Figure 2. Optics schematic detailing FT-IR microscope operation modes. (A) In transmission mode, the sample is illuminated through the bottom objective, and the light passing through the sample is collected by the top objective. (B) In reflection mode, the top objective serves both to illuminate the sample and to collect the reflected light. The bottom objective is not used. Please click here to view a larger version of this figure. 

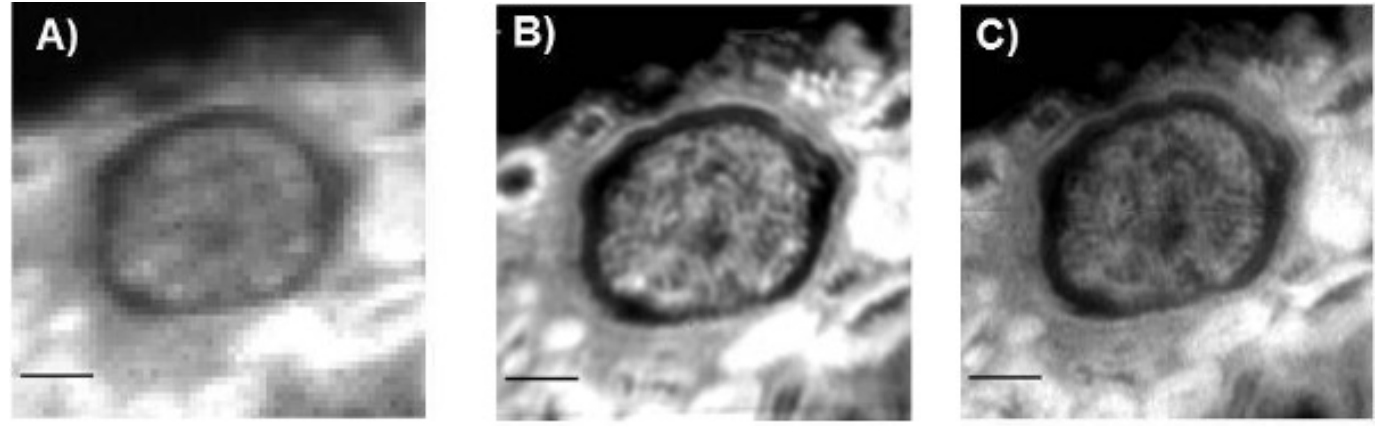

Figure 3. Comparison of different microscope objectives on FT-IR images of a kidney glomerulus at $2,925 \mathrm{~cm}^{-1}$. (A) $15 X$ collecting objective with NA $=0.5(5.5 \times 5.5 \mu \mathrm{m}$ pixel size $)$. (B) $36 \mathrm{X}$ collecting objective with NA $=0.5(2.2 \times 2.2 \mu \mathrm{m}$ pixel size $)$. (C) $74 \mathrm{X}$ collecting objective with $\mathrm{NA}=0.65(1.1 \times 1.1 \mu \mathrm{m}$ pixel size $)$. Scale bar $=50 \mu \mathrm{m}$. Please click here to view a larger version of this figure.
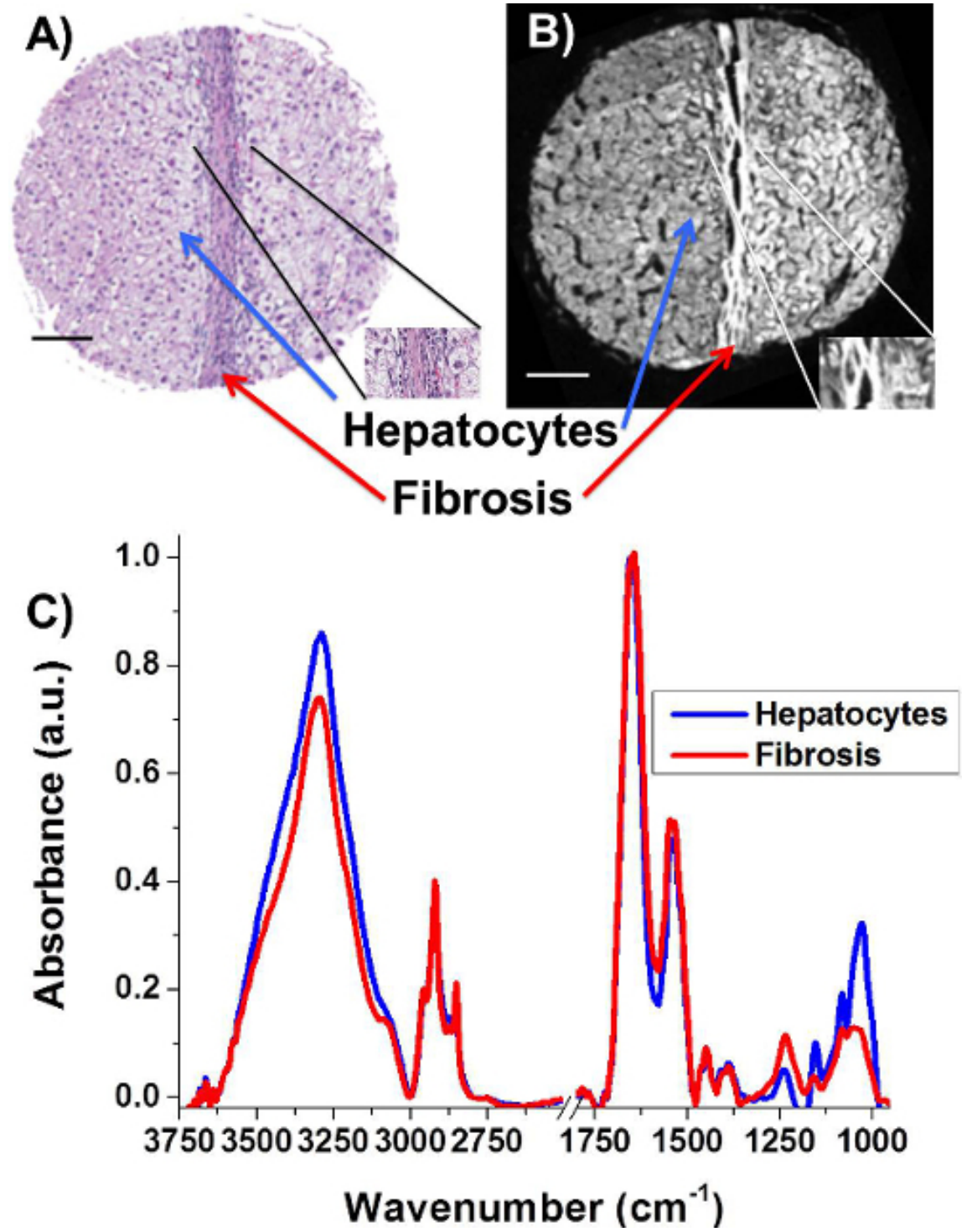

Figure 4. Spectral differences between fibrosis and hepatocytes in a liver core. (A) H\&E stained core from liver biopsy. (B) Image of a serial section core scanned in FT-IR (36X objective setup). (C) Representative spectra of hepatocytes and fibrosis, taken from regions of tissue indicated by arrows in $(\mathbf{A})$ and $(\mathbf{B})$. Scale bar $=100 \mu \mathrm{m}$. Please click here to view a larger version of this figure. 
A)

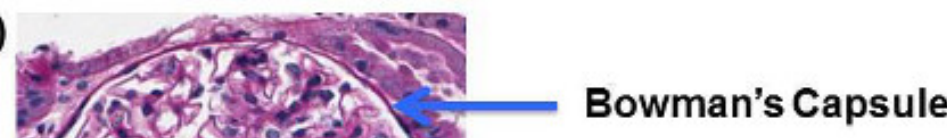

Mesangium

\section{Glomerular Basement Membrane}

B)

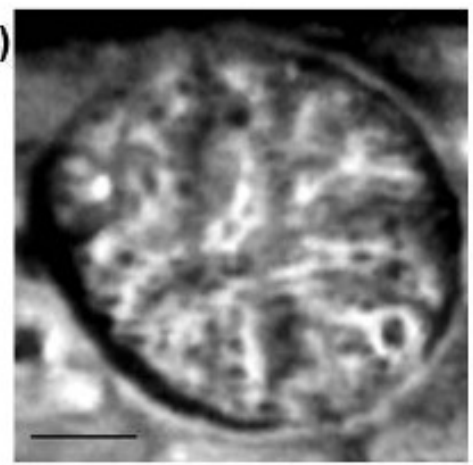

\section{Tubular Basement Membrane}

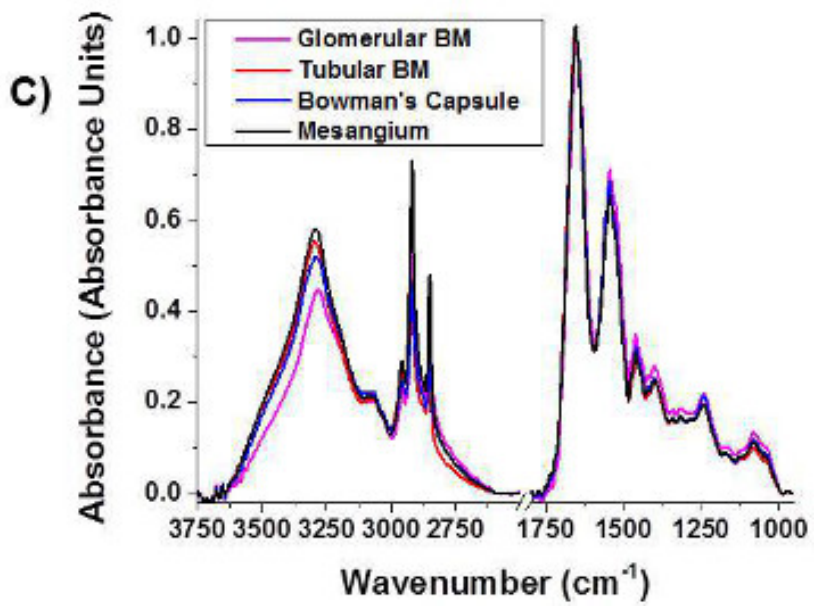

Figure 5. Differentiation of kidney tissue biopsy features through the use of high-definition FT-IR imaging. (A) Periodic acid-Schiff stained section with the features to be extracted labeled. (B) High-definition FT-IR image of the $\mathrm{CH}_{2}$ asymmetric stretching region (36X objective setup) of a serial section of the same tissue. (C) Features labeled in (A) extracted using the FT-IR image in (B) to be able to chemically differentiate the four features of the tissue. Scale bar $=50 \mu \mathrm{m}$. Please click here to view a larger version of this figure.

\section{Discussion}

FT-IR is an emerging modality for label-free biochemical imaging of tissue sections, with the potential to have an important role in improving the current standard of diagnosis in pathology. The current gold standard for pathology requires tissues to be biopsied, fixed in formalin, embedded in paraffin, sectioned multiple times, and stained with multiple stains. A highly trained pathologist has to subjectively visually assess the tissue structure and cellular morphology to determine a diagnosis. Here we show how to collect high-resolution IR images from the same type of sections and discuss some of the computational approaches to examine chemical differences between cell types and disease states.

The critical steps within this protocol are to ensure that the tissues are very carefully focused and that the system is well calibrated to ensure very high quality spectroscopic data. The care when setting up the system is particularly critical when working with high magnification objectives. To aid in troubleshooting, the following list covers some of the potential difficulties encountered;

Problem: Low IR intensity when imaging in reflection. Solution: Check IR slide orientation as the reflective coating may be on the wrong side of the slide.

Problem: Low signal/Red warning sign in Lancer Control. Solution: Cool detectors with LN2. Liquid nitrogen is required for the FPA detectors to function and requires periodically being topped up.

Problem: Velocity error/movement errors. Solution: Reset spectrometer and reduce vibrations. Vibrations will cause the moving mirror in the interferometer to be disturbed.

Problem: Water vapor spikes in data. Solution: Increase purge on system and protect sample from air.

Problem: Invalid centerburst. Solution: Find centerburst again.

Problem: Low flux difference in transmission, even though focused. Solution: Adjust bottom condenser. This will occur as the IR light is not being focused to a point on the sample.

In this paper, we have focused on how to acquire high definition IR images of tissues in either transmission or transflectance mode. The nature of FT-IR imaging, is that there are multiple modifications that can be made to the data acquisition, such as, type of substrate, fixation technique sample thickness, spectral resolution, interferometer mirror speed etc. The effect of these parameters has been discussed in extensive detail recently $4,5,17,51$ 
There are a number of modifications that can be made to the imaging system including imaging in ATR mode A $^{10,24,26}$ and using nanoscale thermal approaches ${ }^{52,53}$ to allow for high resolution IR imaging. The main limitation with high resolution IR imaging is that the tissues must be carefully prepared and thin enough for IR to pass through (typically $4 \mu \mathrm{m}$ thickness). In addition, transmission and reflectance FT-IR imaging requires the samples to be dry due to the absorbance of IR by water. However, FT-IR imaging has significant advantages over other techniques, in that it can very rapidly image large areas of tissue while deriving rich and detailed biochemical information. Other similar techniques that derive biochemical information in a label-free fashion include Raman spectroscopy, however the time of data acquisition is much slower to acquire images. New Raman imaging approaches are emerging including Stimulated Raman scattering (SRS) and Coherent Antistokes Raman scattering (CARS); however, they have access limited spectral range or single frequency imaging.

The advances in speed of data acquisition, spatial resolution, and availability of computational approaches have been of tremendous value in making FT-IR imaging a more feasible approach for translation as a new imaging tool in pathology. The recent advances in spatial resolution have been particularly important for tissue pathology due to key cell types not being resolvable using conventional FT-IR imaging systems. The recent paper by Reddy et al. showed how to model an ideal system to obtain the optimal spatial resolution of an FT-IR imaging system ${ }^{5}$. The kidney tissue example presented in this paper demonstrates the importance of higher spatial resolutions in order to extract biochemical information from glomerular structures (Figure 3 and Figure 5). In the future, new advances in Quantum Cascade Lasers as very bright IR light sources ${ }^{54-57}$, 3D spectral imaging ${ }^{58}$, and breakthroughs in the field of nanoscale IR technologies ${ }^{52,53,59,60}$ hold exciting new avenues of research that may have huge implications in the future of tissue imaging.

We have presented examples of applications in liver and kidney disease where there is a need for additional biochemical information that can be of diagnostic value. The Spectral Pathology Lab in the Department of Pathology at the University of Illinois at Chicago is focused on the translation of IR imaging technologies towards improving disease diagnosis and improved prediction of patient outcome. FT-IR imaging may overcome some of the current limitations in pathology practice where quantitative and objective information is required. In particular, future work is focused on identifying areas in current pathology practice where current techniques fail to provide adequate diagnostic sensitivity or provide limited information. A clear need exists in improving the current practice of pathology and towards giving more information to the pathologist about a patient's disease status, which may be achievable using high-definition FT-IR imaging.

\section{Disclosures}

The authors have nothing to disclose.

\section{Acknowledgements}

We would like to acknowledge the Department of Pathology at the University of Illinois at Chicago for financial support. Histology and visible imaging services were provided by the Research Resources Center - Research Histology and Tissue Imaging Core at the University of Illinois at Chicago established with the support of the Vice Chancellor of Research, in particular we would like to thank Ryan Deaton and Andy Hall for their expertise. We would also like to thank Agilent Technologies, in particular Frank Weston for support and loaning of additional IR lens.

\section{References}

1. Lewis, E. N. et al. Fourier transform spectroscopic imaging using an infrared focal-plane array detector. Anal Chem. 67 (19), $3377-3381$ doi:10.1021/ac00115a003, (1995).

2. Dorling, K. M., \& Baker, M. J. Rapid FTIR chemical imaging: highlighting FPA detectors. Trends Biotechnol. 31 (8), 437-438, doi:10.1016/ j.tibtech.2013.05.008, (2013).

3. Fernandez, D. C., Bhargava, R., Hewitt, S. M., \& Levin, I. W. Infrared spectroscopic imaging for histopathologic recognition. Nat Biotechnol. 23 (4), 469-474, doi:10.1038/nbt1080, (2005).

4. Nasse, M. J. et al. High-resolution Fourier-transform infrared chemical imaging with multiple synchrotron beams. Nat Methods. 8 (5), $413-416$ doi:10.1038/nmeth.1585 (2011).

5. Reddy, R. K., Walsh, M. J., Schulmerich, M. V., Carney, P. S., \& Bhargava, R. High-definition infrared spectroscopic imaging. App/ Spectrosc. 67 (1), 93-105, doi:10.1366/11-06568, (2013).

6. Walsh, M. J., Bhargava, R. Chapter 10; Infrared spectroscopic imaging: an integrative approach to pathology. Nanobiophotonics. McGraw Hill, (2010).

7. Walsh, M. J., Reddy, R. K., \& Bhargava, R. Label-Free Biomedical Imaging With Mid-IR Spectroscopy. leee J Sel Top Quant. 18 (4), 1502-1513, doi:10.1109/Jstqe.2011.2182635 (2012).

8. Matthaus, C. et al. Chapter 10: Infrared and Raman microscopy in cell biology. Methods Cell Biol. 89, 275-308, doi:10.1016/ s0091-679x(08)00610-9, (2008).

9. Walsh, M. J. et al. IR microspectroscopy: potential applications in cervical cancer screening. Cancer Lett. 246 (1-2), 1-11, doi:10.1016/ j.canlet.2006.03.019, (2007).

10. Kazarian, S. G., \& Chan, K. L. ATR-FTIR spectroscopic imaging: recent advances and applications to biological systems. Analyst. 138 (7), 1940-1951, doi:10.1039/c3an36865c, (2013).

11. Sahu, R. K., \& Mordechai, S. Spectral signatures of colonic malignancies in the mid-infrared region: from basic research to clinical applicability. Future Oncol. 6 (10), 1653-1667, doi:10.2217/fon.10.120, (2010).

12. Kendall, C. et al. Vibrational spectroscopy: a clinical tool for cancer diagnostics. Analyst. 134 (6), 1029-1045, doi:10.1039/b822130h, (2009).

13. Steiner, G., \& Koch, E. Trends in Fourier transform infrared spectroscopic imaging. Anal Bioanal Chem. 394 (3), 671-678, doi:10.1007/ s00216-009-2737-5, (2009).

14. Biswas, S., Walsh, M. J., \& Bhargava, R. in Optical Spectroscopy and Computational Methods in Biology and Medicine. Vol. 14 Challenges and Advances in Computational Chemistry and Physics Ch. 16, 475-504, (2014).

15. Bhargava, R. Infrared Spectroscopic Imaging: The Next Generation. App/ Spectrosc. 66 (10), 1091-1120 doi:10.1366/12-06801, (2012). 
16. Malek, K., Wood, B. R., \& Bambery, K. R. in Optical Spectroscopy and Computational Methods in Biology and Medicine. Vol. 14 Challenges and Advances in Computational Chemistry and Physics Ch. 15, 419-473, (2014).

17. Martin, F. L. et al. Distinguishing cell types or populations based on the computational analysis of their infrared spectra. Nat Protoc. 5 (11), 1748-1760, doi:10.1038/nprot.2010.133, (2010).

18. Sommer, A. J., Tisinger, L. G., Marcott, C., \& Story, G. M. Attenuated Total Internal Reflection Infrared Mapping Microspectroscopy Using an Imaging Microscope. Appl Spectrosc. 55 (3), 252-256, doi:10.1366/0003702011951803, (2001).

19. Glassford, S. E., Byrne, B., \& Kazarian, S. G. Recent applications of ATR FTIR spectroscopy and imaging to proteins. Biochim Biophys Acta. 1834 (12), 2849-2858, doi:10.1016/j.bbapap.2013.07.015, (2013).

20. Andanson, J. M., Chan, K. L., \& Kazarian, S. G. High-throughput spectroscopic imaging applied to permeation through the skin. App/ Spectrosc. 63 (5), 512-517, doi:10.1366/000370209788347011, (2009).

21. Kuimova, M. K., Chan, K. L., \& Kazarian, S. G. Chemical imaging of live cancer cells in the natural aqueous environment. App/ Spectrosc. 63 (2), 164-171, doi:10.1366/000370209787391969, (2009).

22. Chan, K. L., \& Kazarian, S. G. Attenuated total reflection-Fourier transform infrared imaging of large areas using inverted prism crystals and combining imaging and mapping. Appl Spectrosc. 62 (10), 1095-1101, doi:10.1366/000370208786049042, (2008).

23. Gajjar, K. et al. Diagnostic segregation of human brain tumours using Fourier-transform infrared and/or Raman spectroscopy coupled with discriminant analysis. Anal Methods. 5, 89-102, doi:10.1039/c2ay25544h, (2012).

24. Holton, S. E., Walsh, M. J., \& Bhargava, R. Subcellular localization of early biochemical transformations in cancer-activated fibroblasts using infrared spectroscopic imaging. Analyst. 136 (14), 2953-2958, doi:10.1039/c1an15112f, (2011).

25. Gulley-Stahl, H. J., Bledsoe, S. B., Evan, A. P., \& Sommer, A. J. The advantages of an attenuated total internal reflection infrared microspectroscopic imaging approach for kidney biopsy analysis. Appl Spectrosc. 64 (1), 15-22 doi:10.1366/000370210792966161, (2010).

26. Walsh, M. J., Kajdacsy-Balla, A., Holton, S. E., \& Bhargava, R. Attenuated total reflectance Fourier-transform infrared spectroscopic imaging for breast histopathology. Vib Spectrosc. 60, 23-28, doi:10.1016/j.vibspec.2012.01.010, (2012).

27. Sreedhar, H. et al.Investigating the Biochemical Progression of Liver Disease Through Fibrosis, Cirrhosis, Dysplasia and Hepatocellular Carcinoma using Fourier Transform Infrared Spectroscopic Imaging. Proc. SPIE 8939, Biomedical Vibrational Spectroscopy VI: Advances in Research and Industry 89390J. doi:10.1117/12.2040408, (2014).

28. Reddy, R. K., \& Bhargava, R. Accurate histopathology from low signal-to-noise ratio spectroscopic imaging data. Analyst. 135 (11), 2818-2825, doi:10.1039/c0an00350f, (2010).

29. Bassan, P. et al. The inherent problem of transflection-mode infrared spectroscopic microscopy and the ramifications for biomedical single point and imaging applications. Analyst. 138 (1), 144-157, doi:10.1039/c2an36090j, (2013).

30. Bassan, P. et al. FTIR microscopy of biological cells and tissue: data analysis using resonant Mie scattering (RMieS) EMSC algorithm. Analyst. 137 (6), 1370-1377, doi:10.1039/c2an16088a, (2012).

31. Bassan, P. et al. Resonant Mie scattering in infrared spectroscopy of biological materials--understanding the 'dispersion artefact'. Analyst. 134 (8), 1586-1593, doi:10.1039/b904808a, (2009).

32. Bassan, P. et al. RMieS-EMSC correction for infrared spectra of biological cells: extension using full Mie theory and GPU computing. $J$ Biophotonics. 3 (8-9), 609-620, doi:10.1002/jbio.201000036, (2010).

33. Bassan, P. et al. Resonant Mie scattering (RMieS) correction of infrared spectra from highly scattering biological samples. Analyst. 135 (2), 268-277, doi:10.1039/b921056c, (2010).

34. Bassan, P. et al. Reflection contributions to the dispersion artefact in FTIR spectra of single biological cells. Analyst. 134 (6), 1171-1175 doi:10.1039/b821349f, (2009).

35. Davis, B. J., Carney, P. S., \& Bhargava, R. Theory of mid-infrared absorption microspectroscopy: II. Heterogeneous samples. Anal Chem. 82 (9), 3487-3499, doi:10.1021/ac902068e, (2010).

36. Davis, B. J., Carney, P. S., \& Bhargava, R. Theory of midinfrared absorption microspectroscopy: I. Homogeneous samples. Anal Chem. 82 (9), 3474-3486, doi:10.1021/ac902067p, (2010).

37. Kohler, A. et al. Estimating and correcting mie scattering in synchrotron-based microscopic fourier transform infrared spectra by extended multiplicative signal correction. Appl Spectrosc. 62 (3), 259-266, doi:10.1366/000370208783759669, (2008).

38. Miljkovic, M., Bird, B., Lenau, K., Mazur, A. I., \& Diem, M. Spectral cytopathology: new aspects of data collection, manipulation and confounding effects. Analyst. 138 (14), 3975-3982, doi:10.1039/c3an00185g, (2013).

39. Miljkovic, M., Bird, B., \& Diem, M. Line shape distortion effects in infrared spectroscopy. Analyst. 137 (17), 3954-3964, doi:10.1039/ c2an35582e, (2012).

40. Bird, B., Miljkovic, M., \& Diem, M. Two step resonant Mie scattering correction of infrared micro-spectral data: human lymph node tissue. $J$ Biophotonics. 3 (8-9), 597-608, doi:10.1002/jbio.201000024, (2010).

41. Mohlenhoff, B., Romeo, M., Diem, M., \& Wood, B. R. Mie-type scattering and non-Beer-Lambert absorption behavior of human cells in infrared microspectroscopy. Biophys J. 88 (5), 3635-3640, doi:10.1529/biophysj.104.057950, (2005).

42. Bambery, K. R., Wood, B. R., \& McNaughton, D. Resonant Mie scattering (RMieS) correction applied to FTIR images of biological tissue samples. Analyst. 137 (1), 126-132, doi:10.1039/c1an15628d, (2012).

43. Kwak, J. T., Reddy, R., Sinha, S., \& Bhargava, R. Analysis of variance in spectroscopic imaging data from human tissues. Anal Chem. 84 (2) 1063-1069, doi:10.1021/ac2026496, (2012).

44. Trevisan, J., Angelov, P. P., Carmichael, P. L., Scott, A. D., \& Martin, F. L. Extracting biological information with computational analysis of Fourier-transform infrared (FTIR) biospectroscopy datasets: current practices to future perspectives. Analyst. 137 (14), 3202-3215, doi:10.1039/c2an16300d, (2012).

45. Trevisan, J. et al. Measuring similarity and improving stability in biomarker identification methods applied to Fourier-transform infrared (FTIR) spectroscopy. J Biophotonics. 7 (3-4), 254-265, doi:10.1002/jbio.201300190, (2014).

46. Bhargava, R., Fernandez, D. C., Hewitt, S. M., \& Levin, I. W. High throughput assessment of cells and tissues: Bayesian classification of spectral metrics from infrared vibrational spectroscopic imaging data. Biochim Biophys Acta. 1758 (7), 830-845, doi:10.1016/ j.bbamem.2006.05.007, (2006).

47. Menze, B. H. et al. A comparison of random forest and its Gini importance with standard chemometric methods for the feature selection and classification of spectral data. BMC bioinformatics. 10, 213, doi:10.1186/1471-2105-10-213, (2009).

48. Kelly, J. G. et al. Biospectroscopy to metabolically profile biomolecular structure: a multistage approach linking computational analysis with biomarkers. J Proteome Res. 10 (4), 1437-1448, doi:10.1021/pr101067u, (2011). 
49. Bird, B. et al. Infrared micro-spectral imaging: distinction of tissue types in axillary lymph node histology. BMC Clin Pathol. 8, 8, doi:10.1186/1472-6890-8-8, (2008).

50. Baker, M. J. et al. Investigating FTIR based histopathology for the diagnosis of prostate cancer. J Biophotonics. 2 (1-2), 104-113, doi:10.1002/jbio.200810062, (2009).

51. Baker, M. J. et al. Using Fourier transform IR spectroscopy to analyze biological materials. Nat Protoc. 9(8), 1771-1791, doi:10.1038/ nprot.2014.110, (2014).

52. Katzenmeyer, A. M., Aksyuk, V., \& Centrone, A. Nanoscale Infrared Spectroscopy: Improving the Spectral Range of the Photothermal Induced Resonance Technique. Anal Chem. 85 (4), 1972-1979, doi:10.1021/ac303620y, (2013).

53. Dazzi, A. et al. AFM-IR: combining atomic force microscopy and infrared spectroscopy for nanoscale chemical characterization. Appl Spectrosc. 66 (12), 1365-1384, doi:10.1366/12-06804, (2012).

54. Kole, M. R., Reddy, R. K., Schulmerich, M. V., Gelber, M. K., \& Bhargava, R. Discrete frequency infrared microspectroscopy and imaging with a tunable quantum cascade laser. Anal Chem. 84 (23), 10366-10372, doi:10.1021/ac302513f, (2012).

55. Vrancic, C. et al. Continuous glucose monitoring by means of mid-infrared transmission laser spectroscopy in vitro. Analyst. 136 (6), 1192-1198, doi:10.1039/c0an00537a, (2011).

56. Yeh, K., Schulmerich, M., \& Bhargava, R. Mid-infrared microspectroscopic imaging with a quantum cascade laser. Proc. SPIE. 8726, NextGeneration Spectroscopic Technologies VI, 87260E, doi:10.1117/12.2015984, (2013).

57. Brandstetter, M., Volgger, L., Genner, A., Jungbauer, C., \& Lendl, B. Direct determination of glucose, lactate and triglycerides in blood serum by a tunable quantum cascade laser-based mid-IR sensor. Appl. Phys. B. 110 (2), 233-239, doi:10.1007/s00340-012-5080-z, (2013).

58. Martin, M. C. et al. 3D spectral imaging with synchrotron Fourier transform infrared spectro-microtomography. Nat Meth. 10 (9), $861-864$, doi:10.1038/nmeth.2596, (2013).

59. Kwon, B. et al. Infrared microspectroscopy combined with conventional atomic force microscopy. Ultramicroscopy. 116, 56-61, doi:10.1016/ j.ultramic.2012.03.007, (2012).

60. Marcott, C. et al. Nanoscale infrared (IR) spectroscopy and imaging of structural lipids in human stratum corneum using an atomic force microscope to directly detect absorbed light from a tunable IR laser source. Exp Dermatol. 22 (6), 419-421, doi:10.1111/exd.12144, (2013). 